\title{
No better solution for antipsychotic treatment with regard to long-term safety and effectiveness
}

\author{
Edward Chia-Cheng Lai, ${ }^{1,2}$ Yea-Huei Kao Yang ${ }^{1,3}$
}

${ }^{1}$ Institute of Clinical Pharmacy and Pharmaceutical Sciences, College of Medicine, National Cheng Kung University, Tainan, Taiwan; ${ }^{2}$ Duke Clinical Research Institute, Duke University School of Medicine, Durham, North Carolina, USA; ${ }^{3}$ Health Outcome Research Center, National Cheng Kung University, Tainan, Taiwan

Correspondence to Dr Edward Chia-Cheng Lai; speedpopo@hotmail.com

\section{WHAT IS ALREADY KNOWN ON THIS TOPIC?}

Using various end points, the comparative effectiveness and safety of antipsychotics in patients with schizophrenia and other psychiatric indications have been demonstrated by a number of experimental and observational studies. ${ }^{1}$ While atypical antipsychotics (AAPs) are indicated more frequently and extensively, their long-term safety in older patients, including the risk of weight gain, metabolic syndrome, cardiovascular and cerebrovascular events, has been a growing concern. ${ }^{2}$

\section{WHAT DOES THIS PAPER ADD?}

- The study indicated lack of effectiveness and a high incidence of side effects across the four commonly prescribed AAPs, namely aripiprazole, olanzapine, quetiapine and risperidone. Caution in the use of these drugs is warranted in middle-aged and older patients.

- The authors designed a pragmatic clinical trial using equipoisestratified randomisation that allowed the patient and the treating psychiatrist to exclude one or two of the study medications for randomisation. This procedure allowed pairwise contrasts of treatments and optimised recruitment resources, increasing the applicability of the findings to real-world clinical management of patients.

\section{LIMITATIONS}

- Patients included had different psychiatric disorders (schizophrenia, mood disorders, dementia or post-traumatic stress disorder) and there were discrepancies between the baseline risks for adverse events. Moreover, as patients included in the study were not incident users, residual effects from patients' previous antipsychotics might have affected the risk estimations.

- The selection bias could not be avoided due to the nature of equipoise-stratified randomisation; for example, patients at high risk of metabolic syndrome were more likely to receive aripiprazole due to clinicians' preference, leading to a potential overestimation of the risk of such population.

- Although the study was designed to follow-up patients for 2 years, in fact, the median duration to discontinuation was only 26 weeks. The high discontinuation rate of antipsychotic treatment may limit the capacity for evaluation of long-term safety.

\section{WHAT NEXT IN RESEARCH?}

Assessments based on reports of outcome by patients and/or their major caregivers would also be valuable to evaluate subjectively perceived health and mental status on antipsychotics treatment. From the perspective of cost-effectiveness, some other antipsychotics would be suitable candidates for future comparative research. ${ }^{3}$

\section{DO THESE RESULTS CHANGE YOUR PRACTICES AND WHY?}

No, because a safe and effective preferred alternative for long-term treatment has not yet been demonstrated. However, as long-term treatment is often necessary for controlling psychotic symptoms, clinicians should always prescribe antipsychotics with caution by monitoring metabolic markers and physiological parameters more frequently, and regardless of the drug selected.

Competing interests None.

doi:10.1136/eb-2014-101801

\section{REFERENCES}

1. Leucht S, Corves C, Arbter D, et al. Second-generation versus first-generation antipsychotic drugs for schizophrenia: a meta-analysis. Lancet 2009;373:31-41.

2. Setoguchi S, Wang PS, Alan Brookhart M, et al. Potential causes of higher mortality in elderly users of conventional and atypical antipsychotic medications. J Am Geriatr Soc 2008;56:1644-50.

3. Lai EC, Chang $\mathrm{CH}, \mathrm{Kao}$ Yang $\mathrm{YH}$, et al. Effectiveness of sulpiride in adult patients with schizophrenia. Schizophr Bull 2013;39:673-83.

ABSTRACT FROM: Jin H, Shih PA, Golshan S, et al. Comparison of longer-term safety and effectiveness of 4 atypical antipsychotics in patients over age 40: a trial using equipoise-stratified randomization. J Clin Psychiatry 2013;74:10-18.

\footnotetext{
Patients/participants 322 adults aged over 40 years with DSM-IVTR-diagnosed schizophrenia, schizoaffective disorder, psychosis associated with mood disorder, post-traumatic stress disorder or dementia, who were either receiving atypical antipsychotics (AAPs) or had AAP prescription proposed by their treating psychiatrist.

Setting The USA; trial dates not reported.

Intervention Aripiprazole, olanzapine, quetiapine or risperidone. Participants were assigned to AAP using equipoise-stratified randomisation. Starting dose and any adjustments were made by the treating psychiatrist.

Patient follow-up Two years (\% follow-up not reported).

Allocation Not reported.

Blinding Single blind (assessors).
}

\section{OUTCOMES}

Time to drug discontinuation Median time to randomised AAP discontinuation was 26 weeks. The proportion of participants who discontinued AAP treatment during follow-up ranged from $78.6 \%$ taking quetiapine to $81.5 \%$ taking aripiprazole; where recorded, reasons for treatment change included adverse effects $(51.6 \%)$ or lack of effectiveness (26.9\%). Participants receiving aripiprazole were significantly less likely to still be on treatment at 6 months follow-up compared to participants receiving olanzapine (26\% vs 58\%).

Metabolic syndrome (incidence at $\mathbf{1}$ year) Eighty-six per cent of patients taking aripiprazole had developed metabolic syndrome compared to $55 \%$ taking olanzapine. There were no significant differences between AAPs for any primary metabolic markers (body mass index, blood pressure, glucose, low-density or high-density lipoproteins or triglycerides).

Adverse events Overall, 50.8\% developed non-serious adverse events (NSAE). Significantly more participants in the quetiapine group developed NSAEs compared to the aripiprazole group (78\% vs $49 \%, p=0.03)$. Significantly more participants receiving olanzapine developed NSAEs compared to participants receiving risperidone ( $73 \%$ vs $46 \%, p=0.04)$. 